\title{
An Unconventional Origin of the Right Colic Artery: A Case
}

\section{Report}

\author{
Elysia Jongue ${ }^{1 *}$, Thomas Suhardja², Ramin Mehidpour ${ }^{2}$, Chris Briggs $^{3}$ \\ and Asiri Arachchi ${ }^{2}$ \\ ${ }^{1}$ College of Medicine and Public Health, Flinders University, Australia \\ Case Report \\ Volume 2 Issue 2 \\ Received Date: June 21, 2018 \\ ${ }^{2}$ Department of Surgery, Monash Medical Centre, Australia \\ Published Date: July 04, 2018
}

${ }^{3}$ Professor, Department of Anatomy and Neuroscience, The University of Melbourne, Australia

*Corresponding author: Elysia Jongue, Flinders University, Building 4a, Nightingdale Rd, Royal Darwin Hospital Campus, Tiwi, Northern Territory, 0810 Australia, Email: elysiajongue@gmail.com

\begin{abstract}
Anatomical variations of the origin of the right colic artery exist. Routine dissection of a cadaver revealed that the right colic artery arose from the proximal middle colic artery prior to the latter's branching. Hence, a common stem of the right colic artery and the middle colic artery existed, where this common stem arose directly from the superior mesenteric artery. Limited documentation of the specific right colic-middle colic common branch variation, displays an opportunity within the literature for further case reports and research into this variation to revise incidence rates of this right colic artery origin that will subsequently influence medical education and surgical procedures and ultimately, patient treatment and care.
\end{abstract}

Keywords: Right colic artery; Middle colic artery; Superior mesenteric artery; Common trunk; Colic artery origin; Anatomical variation

\section{Introduction}

Between 1759 and 1766, von Haller established the fundamental outline of colonic blood supply accepted by anatomists, revealing that the blood supply of the colon is primarily delivered by the superior and inferior mesenteric arteries [1]. Particularly, the arterial blood source of the midgut is delivered by branches of the superior mesenteric artery including the ileocolic, right colic and middle colic arteries. These arteries classically arise independently from the right side of the superior mesenteric artery and diverge laterally to supply the distal midgut. The ileocolic artery arises near the root of the mesentery, the right colic artery emerges between the ileocolic and middle colic arteries to run across the right psoas muscle, and the middle colic artery arises at the lower border of the pancreas [2] (Figure 1).

Variations in the classical origin of the vasculature exist which include anomalies of the right colic artery origin. Typically originating directly from the superior mesenteric artery, the right colic artery proceeds laterally towards the ascending colon, separating into a descending branch that anastomoses with the superior 
branch of the ileocolic artery, as well as an ascending branch that anastomoses with the right branch of the superior mesenteric artery [2]. Smaller arterial branches of the inferior and superior divisions of the right colic artery go on to supply the distal two-thirds of the ascending colon and the hepatic flexure [3]. Occasionally, the right colic artery's parent vessel may not be the superior mesenteric artery, instead sharing a common trunk with a neighbouring artery before dividing into its branches to supply the colon, or being absent altogether [4].

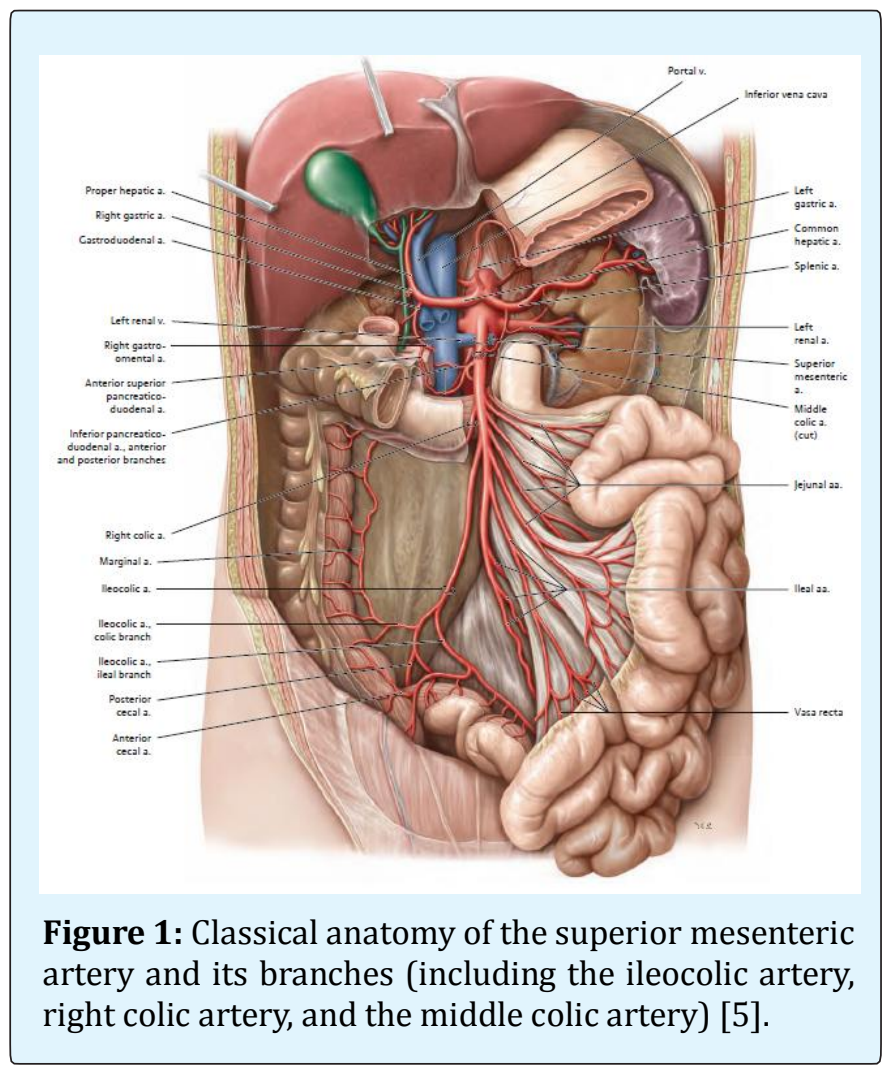

Our work articulates an unconventional origin of the right colic artery, the incidence of this variation in the current literature, as well as the significance of this morphology within a clinical context.

\section{Case Report}

Routine dissection of the abdominal region of a cadaver revealed an anatomical variation in the origin of the right colic artery. This right colic artery branched from the root of the middle colic artery (Figure 2), and hence the right and middle colic arteries shared a common trunk that arose from the superior mesenteric artery. The right and middle colic arteries continued distally to supply their typical respective areas of the midgut. The ileocolic artery maintained its classical origin where it emerges directly from the superior mesenteric artery.

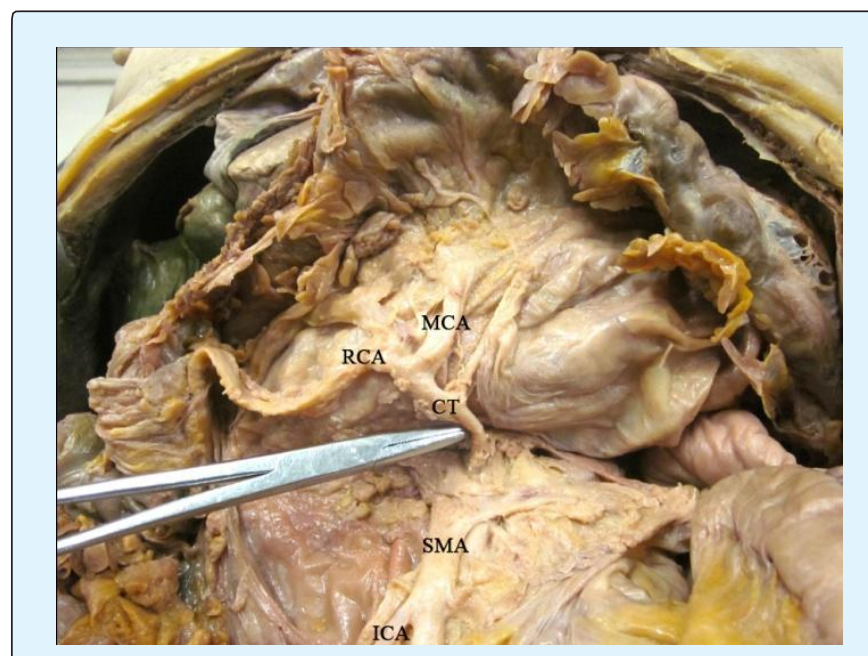

Figure 2: A common trunk shared between the right colic artery and middle colic artery. (SMA: superior mesenteric artery; ICA: ileocolic artery; RCA: right colic artery; MCA: middle colic artery; CT: common trunk of right colic artery and middle colic artery).

\section{Discussion}

Deviations from the classical origin of the right colic artery are acknowledged with differing degrees of detail throughout the current medical literature. Lee et al. (2016), Kuzu et al. (2017), and Murono et al. (2016), establish variability of the right colic artery origin by commenting exclusively on the incidence of the artery arising from its classical origin with values varying from $10.7 \%$ [6] to $33.34 \%$ [7]. Although these authors demonstrate right colic artery variations, other origins of the right colic artery are not explored.

The work of Alsabilah, et al. (2017), Steward and Rankin (1933), and Garcia-Ruiz (1996) further enhance the knowledge of right colic artery variation in that they document the variation in the place of origin. Specifically, their texts communicate cases of the right colic artery originating from the middle colic artery, however do not further define the place of origin along the middle colic artery - whether the origin be from the proximal middle colic artery prior to its branching, or from a branch of the middle colic artery. 
Few reports in the literature communicate cases in which a common trunk for the right colic artery and the middle colic artery exists, further extending knowledge of the variation of right colic artery from the middle colic artery. Haywood, et al. (2016), Ignjatovic, et al. (2007), Michels, et al. (1965), Gamo, et al. (2016), and Haywood, et al. (2017) note cases of a common stem shared by the right and middle colic arteries, branching from the superior mesenteric artery - alternately described as a right colic artery arising from the proximal middle colic artery prior to its branching into the right and left branches of the middle colic arteries. The incidences of the right colic-middle colic common stem extended from $3.33 \%$ [8] to $52 \%$ [9]. Our literature search revealed the work of Haywood, et al. (2017) to be the only systematic review of the variations of right colic artery origin, their paper reviewing the rate of incidence of the right colic artery origin and reporting a revised incidence of $27.7 \%$ [4].

Considering the varying incidence rate of a common trunk of the right and middle colic arteries, additional research is required to provide further data of this anatomical anomaly to allow subsequent systematic reviews to confirm the rate of incidence of the common trunk shared between the right and middle colic arteries. Our case report contributes to the literature in that it provides further data on the right colic-middle colic common trunk variation, where current research is limited. Our case also aims to promote greater recognition of the variation to potentially encourage additional research and analysis into the variation, ultimately allowing for an incidence rate of this particular variation of the right colic artery, to be approximated with greater precision [10-14].

A reviewed incidence rate of the right colic-middle colic common stem anomaly will be valuable within a clinical context, particularly in right-sided colonic surgical procedures - knowledge of the variation promotes greater accuracy in identification allowing opportunity to revise surgical techniques as appropriate, enhancing patient outcomes.

\section{Conclusion}

A common branch shared between the right colic artery and the middle colic artery depicts an unusual variation from the classical origin of the right colic artery that typically arises directly from the superior mesenteric artery. We report a case of this anatomical variation that will contribute to refining the incidence of variations of the right colic artery in future systematic studies, while also furthering understanding of colic artery variations that will be pertinent in education and surgery to allow appropriation of procedural techniques in theatre to suit individual cases.

\section{References}

1. Steward J, Rankin FW (1933) Blood supply of the large intestine: its surgical considerations. Arch Surg 26(5): 843-891.

2. Sinnatamby C (2011) Last's Anatomy. Elsevier Health Sciences.

3. Pal A, Batra AS, Kaur J, Kaur M (2014) Variations in origin of right colic artery supplying colon. J Surg 2: 2319-387514.

4. Haywood M, Molyneux C, Mahadevan V, Srinivasaiah N (2017) Right colic artery anatomy: a systematic review of cadaveric studies. Tech Coloproctol 21(12): 937-943.

5. Gilroy AM, MacPherson BR (2016) Atlas of Anatomy, New York: Thieme Medical Publishers.

6. Garcia-Ruiz A, Milsom JW, Ludwig KA, Marchesa P (1996) Right colonic arterial anatomy, Implications for laparoscopic surgery. Dis Colon Rectum 39(8): 906-911.

7. Murono K, Kawai K, Ishihara S, Otani K, Yasuda K, et al. (2016) Evaluation of the vascular anatomy of the right-sided colon using three-dimentional computed tomography angiography: a single-center study of 536 patients and a review of the literature. Int J Colorectal Disase 31(9): 1633-1638.

8. Ignjatovic D, Sund S, Stimec B, Bergamaschi R (2007) Vascular relationships in right colectomy for cancer: clinical implications. Tech Coloproctol 11(3): 247-250.

9. Michels NA, Siddharth P, Kornblith PL, Parke WW (1965) The variant blood supply to the descending colon, rectosigmoid and rectum based on 400 dissections. Its importance in regional resections: a review of medical literature. Dis Colon Rectum 8: 251278.

10. Haywood M, Molyneux C, Mahadevan V, Lloyd J, Srinivasaiah N (2016) The right colic artery: an anatomical demonstration and its relevance in the 


\section{Journal of Human Anatomy}

laparoscopic era. Ann R Coll Surg Engl 98(8): 560-563.

11. Lee SJ, Park SC, Kim MJ, Sohn DK, Oh JH (2016) Vascular anatomy in laparoscopic colectomy for right colon cancer. Dis Colon Rectum 59(8): 718-724.

12. Kuzu MA, Ismail E, Celik S, Sahin MF, Guner MA, et al. (2017) Variations in the vascular anatomy of the right colon and implications for right-sided colon surgery. Dis Colon \& Rectum 60(3): 290-298.
13. Alsabilah J, Kim W, NK K (2017) Vascular structures of the right colon: incidence and variations with their clinical implications. Scand J Surg 106(2): 107-115.

14. Gamo E, Jimenez C, Pallares E, Simon C, Valderrama F, et al. (2016) The superior mesenteric artery and the variations of the colic patterns. A new anatomical and radiological classification of the colic arteries. Surg Radiol Anat 38(5): 519-527.

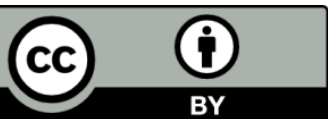

